\title{
Low-Distortion Embeddings of Trees ${ }^{\star}$
}

\author{
Robert Babilon, Jiří Matoušek, Jana Maxová, and Pavel Valtr \\ Department of Applied Mathematics and \\ Institute for Theoretical Computer Science (ITI) \\ Charles University, Malostranské nám. 25, \\ 11800 Prague, Czech Republic, \\ \{babilon, matousek, jana, valtr\}@kam.ms.mff.cuni.cz
}

\begin{abstract}
We prove that every tree $T=(V, E)$ on $n$ vertices can be embedded in the plane with distortion $O(\sqrt{n})$; that is, we construct a mapping $f: V \rightarrow \mathbf{R}^{2}$ such that $\rho(u, v) \leq\|f(u)-f(v)\| \leq O(\sqrt{n}) \cdot \rho(u, v)$ for every $u, v \in V$, where $\rho(u, v)$ denotes the length of the path from $u$ to $v$ in $T$ (the edges have unit lengths). The embedding is described by a simple and easily computable formula. This is asymptotically optimal in the worst case. We also prove several related results.
\end{abstract}

\section{Introduction}

Embeddings of finite metric spaces into Euclidean spaces or other normed spaces that approximately preserve the metric received considerable attention in recent years. Numerous significant results have been obtained in the 1980s, mainly in connection with the local theory of Banach spaces. Later on, surprising algorithmic applications and further theoretical results were found in theoretical computer science; see, for example, 2, 1], 4, [6, and references therein.

Here we consider only embeddings into the spaces $\mathbf{R}^{d}$ with the Euclidean metric. The quality of an embedding is measured by the distortion. Let $(V, \rho)$ be a finite metric space. We say that a mapping $f: V \rightarrow \mathbf{R}^{d}$ has distortion at most $D$ if there exists a real number $\alpha>0$ such that for all $u, v \in V$,

$$
\alpha \cdot \rho(u, v) \leq\|f(u)-f(v)\| \leq D \cdot \alpha \cdot \rho(u, v) .
$$

This definition permits scaling of all distances in the same ratio $r$, in addition to the distortion of the individual distances by factors between 1 and $D$. Since the image in $\mathbf{R}^{d}$ can always be re-scaled as needed, we can choose the factor $\alpha$ at our convenience.

We study embeddings with the dimension $d$ fixed, and mainly the case $d=$ 2, i.e. embeddings into the plane. A straightforward volume argument shows that the distortion required to embed into $\mathbf{R}^{d}$ the $n$-point metric space with all distances equal to 1 is at least $\Omega\left(n^{1 / d}\right)$. In [5], it was proved that general metric spaces may require even significantly bigger distortions; namely, for every

\footnotetext{
* The research was supported by project LN00A056 of the Ministry of Education of the Czech Republic and by Charles University grants No. 158/99 and 159/99.
} 
fixed $d$, there are $n$-point metric spaces that need $\Omega\left(n^{1 /\lfloor(d+1) / 2\rfloor}\right)$ distortion for embedding into $\mathbf{R}^{d}$.

Better upper bounds can be obtained for special classes of metric spaces. A metric $\rho$ on a finite set $V$ is called a tree metric if there is a tree $T=(V, E)$ (in the graph-theoretic sense) and a weight function $w: E \rightarrow(0, \infty)$ such that $\rho(u, v)$ is the length of the path connecting the vertices $u$ and $v$ in $T$, where the length of an edge $e \in E$ is $w(e)$. It was conjectured in [5] that, for fixed $d$, all $n$-point tree metrics can be embedded into $\mathbf{R}^{d}$ with distortion $O\left(n^{1 / d}\right)$. If true, this is best possible, as the example of a star with $n-1$ leaves and unit-length edges shows (a volume argument applies). Gupta [3] proved the somewhat weaker upper bound $O\left(n^{1 /(d-1)}\right)$.

Here we make a step towards establishing the conjecture. We deal with the planar case, where the gap between the lower bound of $\sqrt{n}$ and Gupta's $O(n)$ upper bound is the largest (in fact, the $O(n)$ upper bound holds even for general

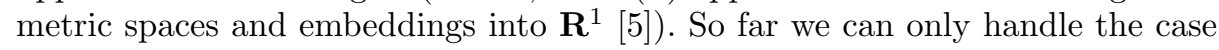
of unit-length edges.

Theorem 1. Every $n$-vertex tree with unit-length edges, considered as a metric space, can be embedded into $\mathbf{R}^{2}$ with distortion $O(\sqrt{n})$. The embedding is described by a simple explicit formula and can be computed efficiently.

Gupta's result actually states that if the considered tree has at most $\ell$ leaves, then an embedding into $\mathbf{R}^{d}$ with distortion $O\left(\ell^{1 /(d-1)}\right)$ is possible. We show that here the dependence on $\ell$ cannot be improved. Let $F_{\ell, m}$ (the fan with $\ell$ leaves and path length $m$ ) denote the tree consisting of $\ell$ paths of length $m$ each glued together at a common vertex (root):

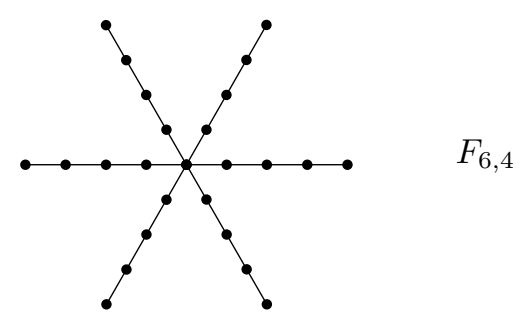

Proposition 1. For every fixed $d \geq 2$ and every $m, \ell \geq 2$ every embedding of $F_{\ell, m}$ into $\mathbf{R}^{d}$ requires distortion $\Omega\left(\ell^{1 /(d-1)}\right)$ if $\ell \leq m^{\frac{d(d-1)}{d+1}}$ and distortion $\Omega\left(\ell^{1 / d} m^{1 /(d+1)}\right)$ if $\ell \geq m^{\frac{d(d-1)}{d+1}}$.

Theorem 1 provides embeddings which are optimal in the worst case, i.e. there are trees for which the distortion cannot be asymptotically improved. But optimal or near-optimal embeddings of special trees seem to present interesting challenges, and sometimes low-distortion embeddings are aesthetically pleasing and offer a good way of drawing the particular trees. We present one interesting example concerning the fan $F_{\sqrt{n}, \sqrt{n}}$. Here the "obvious" embedding as in the above picture, as well as the embedding from Theorem 10 yield distortions 
$O(\sqrt{n})$. In Section 4 we describe a somewhat surprising better embedding, with distortion only $O\left(n^{5 / 12}\right)$. This is already optimal according to Proposition 1 .

Several interesting problems remain open. The obvious ones are to extend Theorem 1 to higher dimensions and/or to trees with weighted edges. Another, perhaps more difficult, question is to extend the class of the considered metric spaces. Most significantly, we do not know an example of an $n$-vertex planar graph (with weighted edges) whose embedding into $\mathbf{R}^{2}$ would require distortion larger than about $\sqrt{n}$. If it were possible to show that all planar-graph metrics can be embedded into the plane with $o(n)$ distortion, it would be a neat metric condition separating planar graphs from non-planar ones, since suitable $n$-vertex subdivisions of any fixed non-planar graph require $\Omega(n)$ distortion [5].

\section{Proof of Theorem 1}

Notation. Let $T$ be a tree (the edges have unit lengths) and let $\rho$ be the shortest-path metric on $V=V(T)$. One vertex is chosen as a root. The height $h(v)$ of a vertex $v \in V$ is its distance to the root. Let $\pi_{v}$ denote the path from a vertex $v$ to the root.

For every vertex we fix a linear (left-to-right) ordering of its children. This defines a partial ordering $\preceq$ on $V$ : we have $u \prec v$ iff $u \notin \pi_{v}, v \notin \pi_{u}$, and $\pi_{v}$ goes right of $\pi_{u}$ at the vertex where $\pi_{u}$ and $\pi_{v}$ branch. We define

$$
\operatorname{sgn}_{v}(u)=\left\{\begin{array}{cl}
0 & \text { if } u \in \pi_{v} \text { or } v \in \pi_{u} \\
+1 & \text { if } u \prec v \\
-1 & \text { if } v \prec u
\end{array}\right.
$$

Further we define $a_{v}(u)$ as the distance of $v$ to the nearest common ancestor of $u$ and $v$, and $\ell(v)=|\{u \in V(T): u \prec v\}|$.

Construction. Our construction resembles Gupta's construction 3 to some extent, but we needed several new ideas to obtain significantly better distortion.

First we describe the idea of the contruction.

The embedded tree is placed in some angle; the root of the tree is placed in the vertex of the angle. In the first step we put vertices of height $h$ on the horizontal line $y=2 \sqrt{n} h$. Our angle is divided into subangles which correspond to subtrees from the root and whose sizes are proportional to the number of vertices in the subtrees. The vertices with height 1 are placed on the first horizontal line in the middle of the corresponding subangles. We continues in the same way for every subtree, with each subangle translated so that its vertex lies at the root of the subtree.

In the second (and last) step we raise the $y$-coordinates of the vertices by appropriate amounts between 0 and $\sqrt{n}$. 


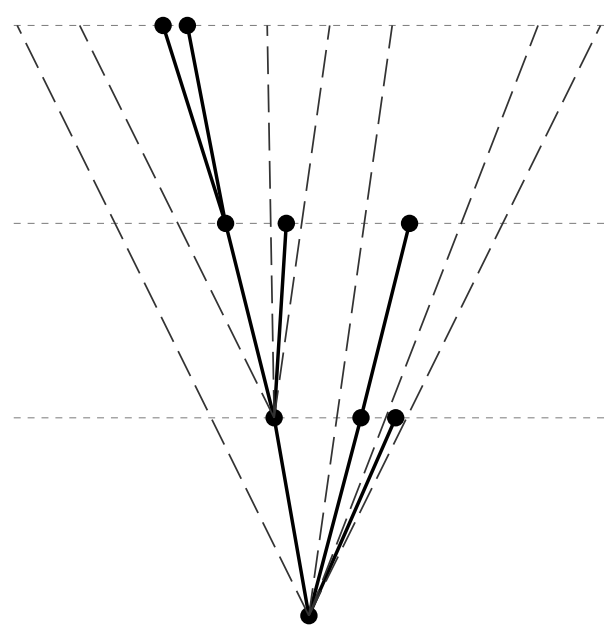

First step

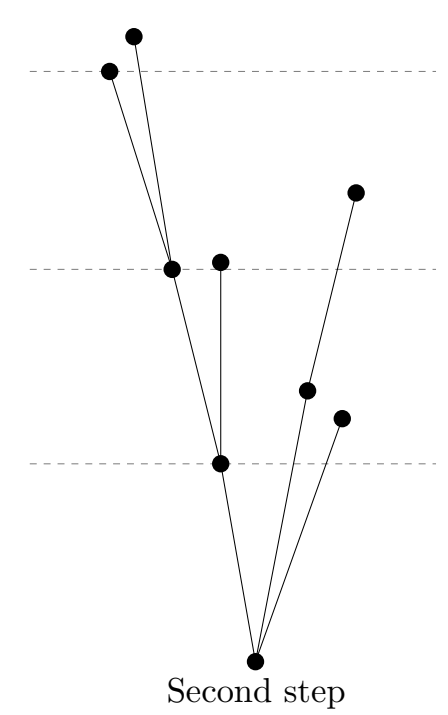

Second step

Now we describe the embedding more formally; actually we use a slightly modified embedding for which the formulas are quite simple.

First we define an auxiliary function $k(v)$ :

$$
k(v)=\sum_{w \in V} \operatorname{sgn}_{v}(w) a_{v}(w) .
$$

The embedding $f: V \rightarrow \mathbf{R}^{2}$ is now defined by $f(v)=(x(v), y(v))$, where

$$
\begin{aligned}
& x(v)=n^{-1 / 2} k(v) \\
& y(v)=2 \sqrt{n} h(v)+(\ell(v) \bmod \sqrt{n}) .
\end{aligned}
$$

Distortion. We now estimate the distortion of the embedding defined above. First we show that the maximum expansion of the tree distance of any two vertices is at most $O(\sqrt{n})$. By the triangle inequality, it suffices to consider two vertices connected by an edge. So let $u$ be the father of $v$ in $T$; it suffices to verify that $\|f(u)-f(v)\|=O(\sqrt{n})$. We clearly have $|y(u)-y(v)| \leq 3 \sqrt{n}$, and so it suffices to prove that $|x(u)-x(v)|=O(\sqrt{n})$, which will follow from $|k(u)-k(v)| \leq n$. We have

$$
|k(v)-k(u)| \leq \sum_{w \in V(T)}\left|\operatorname{sgn}_{u}(w) a_{u}(w)-\operatorname{sgn}_{v}(w) a_{v}(w)\right| .
$$

We always have $\left|a_{u}(w)-a_{v}(w)\right| \leq 1$, as $\rho(u, v)=1$. Moreover, it cannot happen that $\operatorname{sgn}_{u}(w)=-\operatorname{sgn}_{v}(w)$, and so the contribution of each $w$ to the above sum is at most 1 . Therefore $|k(u)-k(v)| \leq n$ as claimed.

Next, we are going to prove that $\|f(u)-f(v)\|=\Omega(\rho(u, v))$ for every $u, v \in$ $V$; this will finish the proof of Theorem[1. 
First we consider the situation when $h(u)=h(v)$; this is the main part of the proof, and the case where $u$ and $v$ have different levels will be an easy extension of this. So let $h(u)=h(v)$ and $a=a_{v}(u)$, and assume that $u \prec v$.

Lemma 1. $\ell(v)-\ell(u) \geq a$

This is because all the vertices on $\pi_{u}$ not lying on $\pi_{v}$ are counted in $\ell(v)$ but not in $\ell(u)$.

Corollary 1. If $\ell(v)-\ell(u)<\frac{1}{2} \sqrt{n}$ then $|y(u)-y(v)| \geq \frac{1}{2} \rho(u, v)$.

Indeed, we have $|y(u)-y(v)|=|(\ell(u) \bmod \sqrt{n})-(\ell(v) \bmod \sqrt{n})| \geq \mid \ell(u)-$ $\ell(v) \mid \geq a=\frac{1}{2} \rho(u, v)$.

Thus, the difference in the $y$-coordinate takes care of $u$ and $v$ whenever $\ell(v)-\ell(u) \leq \frac{1}{2} \sqrt{n}$. Next, we need to show that if this is not the case then the $x$-coordinate takes care of $u$ and $v$.

Lemma 2. $k(v)-k(u) \leq[\ell(v)-\ell(u)] \cdot a$.

Corollary 2. If $\ell(v)-\ell(u) \geq \frac{1}{2} \sqrt{n}$ then $x(v)-x(u)=n^{-1 / 2}[\ell(v)-\ell(u)] \cdot a \geq$ $\frac{1}{2} a \geq \frac{1}{4} \rho(u, v)$.

Combining this corollary with Corollary 1 yields $\|f(u)-f(v)\|=\Omega(\rho(u, v))$ for all $u, v \in V$ with $h(u)=h(v)$.

Proof of Lemma 2, We have $k(v)-k(u)=\sum_{w \in V} t_{u, v}(w)$, where $t_{u, v}(w)=$ $\operatorname{sgn}_{v}(w) a_{v}(w)-\operatorname{sgn}_{u}(w) a_{u}(w)$. First we check that $t_{u, v} \geq 0$ for all $w$. We always have $\operatorname{sgn}_{v}(w) \geq \operatorname{sgn}_{u}(w)$, and so the only case which might cause trouble is $\operatorname{sgn}_{v}(w)=\operatorname{sgn}_{u}(w)=1$. In this case $w \prec u \prec v$, and it is easy to check that then $a_{v}(w) \geq a_{u}(w)$, which shows $t_{u, v} \geq 0$ in all cases.

Next, we verify that if $w$ contributes 1 to $\ell(v)-\ell(u)$, which means $w \prec v$ and $w \nprec u$, then $t_{u, v}(w) \geq a$. We distinguish two cases for such $w$ : either $u \prec w \prec v$ or $w$ and $u$ lie on a common path to the root, i.e. $w \in \pi_{u}$ or $u \in \pi_{w}$.

In the first case, $u \prec w \prec v, \operatorname{sgn}_{u}(w)=-1$ and $\operatorname{sgn}_{v}(w)=1$, and so $t_{u, v}(w)=a_{u}(w)+a_{v}(w)$. It is easy to see that if $u$ and $v$ are vertices with $a_{u}(v)=a_{v}(u)=a$ then $\max \left(a_{u}(w), a_{v}(w)\right) \geq a$ for all $w \in V$ and so $t_{u, v}(w) \geq a$ in this case.

In the second case, we have $\operatorname{sgn}_{v}(w)=1$ and $\operatorname{sgn}_{u}(w)=0$, and so $t_{u, v}(w)=$ $a_{v}(w)=a$, since the nearest common ancestor of $u$ and $v$ is the same as the nearest common ancestor of $w$ and $v$. The proof of Lemma 2 is complete.

Claim. If two vertices $u, v$ are on different levels, then

$$
\|f(v)-f(u)\| \geq \frac{1}{12 \sqrt{2}} \rho(u, v) .
$$

Proof. Without loss of generality, suppose that $h(u)<h(v)$ and $u \prec v$. Set $a:=a_{u}(v)$, and let $w$ be the vertex on the path from $u$ to $v$ with $h(w)=h(u)$, $\rho(u, w)=2 a$. 
If $\rho(u, v)>3 a$ then $\|f(v)-f(u)\| \geq y(v)-y(u) \geq(\rho(u, v)-2 a) \sqrt{n} \geq$ $\frac{\sqrt{n}}{3} \rho(u, v) \geq \frac{1}{3} \rho(u, v)$.

If $\rho(u, v) \leq 3 a$ and $a<4 \sqrt{n}$ then $\|f(v)-f(u)\| \geq \sqrt{n}>a / 4 y(v)-y(u) \geq$ $\frac{1}{12} \rho(u, v)$.

Finally, suppose that $\rho(u, v) \leq 3 a$ and $a \geq 4 \sqrt{n}$. Since the $y$-axis and the extension of any edge span an angle smaller than $45^{\circ}$, the path from $f(w)$ to $f(v)$ lies in the cone bounded by the two halflines emanating from $f(w)$ diagonally upwards and spaning the angle $45^{\circ}$ with the $y$-axis. The point $f(u)$ lies at distance at least $\frac{1}{\sqrt{2}}(x(w)-x(u)+y(w)-y(u))>\frac{1}{\sqrt{2}}(x(w)-x(u)-\sqrt{n}) \geq$ $\frac{1}{\sqrt{2}}(a / 2-\sqrt{n}) \geq \frac{1}{\sqrt{2}} \frac{a}{4} \geq \frac{1}{12 \sqrt{2}} \rho(u, v)$ from this cone (in the second inequality we used Corollary 2). Thus, $\|f(v)-f(u)\|>\frac{1}{12 \sqrt{2}} \rho(u, v)$ in this case.

\section{Proof of Proposition 1}

Let $F_{\ell, m}$ be defined as in Section[1, that is, $F_{\ell, m}$ denotes the tree consisting of $\ell$ paths of length $m$ glued at the root $r$. We show that every embedding of $F_{\ell, m}$ into $\mathbf{R}^{d}$ requires distortion $\Omega\left(\ell^{1 /(d-1)}\right)$ if $\ell \leq m^{\frac{d(d-1)}{d+1}}$ and distortion $\Omega\left(\ell^{1 / d} m^{1 /(d+1)}\right)$ if $\ell \geq m^{\frac{d(d-1)}{d+1}}$.

Let $f: V\left(\dot{F}_{\ell, m}\right) \rightarrow \mathbf{R}^{d}$ be any non-contracting embedding with distortion $D$ (i.e. for all $u, v \in V, \rho(u, v) \leq\|f(u)-f(v)\| \leq D \cdot \rho(u, v))$. We choose a real number $R$ such that there are $\ell / 2$ of the images of endvertices of $F_{\ell, m}$ contained inside the ball $B$ of radius $R$ around $f(r)$ or on its boundary and there are $\ell / 2$ of them otside the ball or on its boundary. Let $S$ denote the sphere of radius $R$ around $f(r)$ (i.e. the boundary of $B$ ). Let $P_{1}, \ldots, P_{\ell / 2}$ denote the $\ell / 2$ paths for which the images of their endvertices are not contained in $B$.

Each $f\left(P_{i}\right)$ intersects $S$ at least once (by $f\left(P_{i}\right)$ we mean $f\left(V\left(P_{i}\right)\right)$ together with straight lines between any $f(u), f(v)$ such that $\left.\{u, v\} \in E\left(P_{i}\right)\right)$. Let $m_{i}$ denote the number of vertices on $f\left(P_{i}\right)$ inside $B$ before the first intersection with $S$. We put $\mu=\min \left\{m_{i} \mid i=1, \ldots, \ell / 2\right\}$. Since $\ell / 2$ of the endvertices are contained in $B$, we immediately have, by a volume argument, $R=\Omega\left(m \ell^{1 / d}\right)$. From the choice of $\mu$ it follows that $R \leq D \mu$.

Let $P$ be a spherical shell of width $D$ around $B$; that is

$$
P=B(f(r), R+D) \backslash \operatorname{int} B(f(r), R) .
$$

At least $\ell / 2$ vertices (one from each path $P_{i}, i=1, \ldots, \ell / 2$ ) are contained in $P$. We consider a ball of radius $\mu$ around each of these vertices. These balls are disjoint (because the tree-distance between any two of the vertices is at least $2 \mu$ ) and they are contained in $\mu$-neighbourhood of $P$ (see the picture). Therefore we have, for a suitable constant $c>0$,

$$
R^{d-1}(D+2 \mu) \geq c \ell \mu^{d} .
$$

Thus there are two possibilities: either $R^{d-1} D=\Omega\left(\ell \mu^{d}\right)$ or $R^{d-1} \mu=\Omega\left(\ell \mu^{d}\right)$.

If $R^{d-1} D=\Omega\left(\ell \cdot \mu^{d}\right)$, then $D^{d+1}=\Omega(\ell \cdot R)$, since $R \leq D \mu$. And since $R=\Omega\left(m \ell^{1 / d}\right)$ we have $D^{d+1}=\Omega\left(\ell \cdot m \cdot \ell^{1 / d}\right)$. Thus $D=\Omega\left(\overline{\ell^{1 / d}} \cdot m^{1 /(d+1)}\right)$. If 


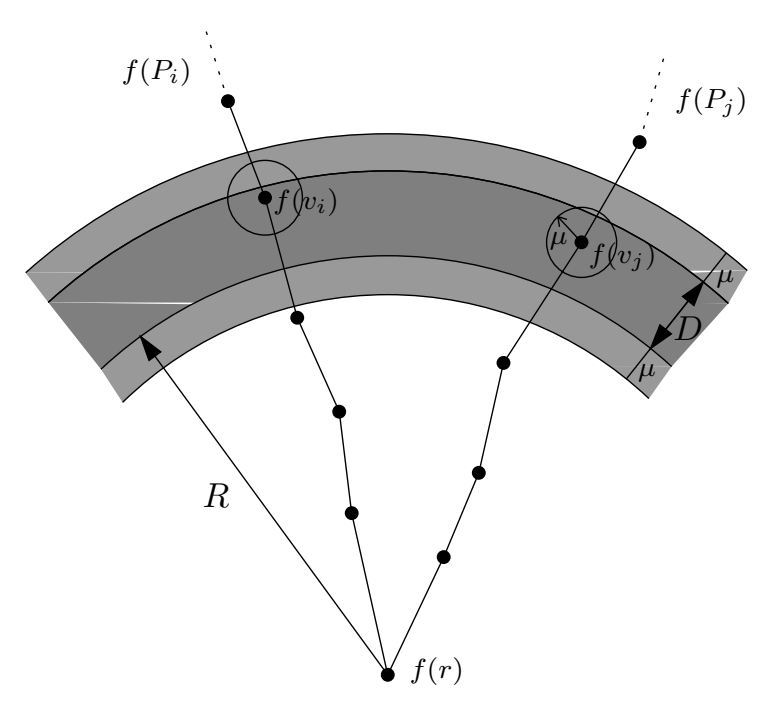

Fig. 1. Illustration to the proof of Proposition 1

$R^{d-1} \mu=\Omega\left(\ell \cdot \mu^{d}\right)$, then since $R \leq D \mu$, we immediately have $D=\Omega\left(\ell^{1 /(d-1)}\right)$. Thus we know that $D \geq \min \left\{\Omega\left(\ell^{1 / d} \cdot m^{1 /(d+1)}\right), \Omega\left(\ell^{1 /(d-1)}\right)\right\}$. It means that $D=\Omega\left(\ell^{1 / d} \cdot m^{1 /(d+1)}\right)$ if $\ell \geq m^{\frac{d(d-1)}{d+1}}$ and $D=\Omega\left(\ell^{1 /(d-1)}\right)$ if $\ell \leq m^{\frac{d(d-1)}{d+1}}$.

\section{A Low-Distortion Embedding of a Subdivided Star}

In this section we describe an asymptotically optimal embedding of $F_{\sqrt{n}, \sqrt{n}}$ into $\mathbf{R}^{2}$, with distortion $O\left(n^{5 / 12}\right)$. The other $F_{\ell, m}$ can be embedded similarly, with distortion as in Proposition 1

The embedding is sketched in Fig 2. The whole tree is embedded into the shaded trapezoids (there are $n^{1 / 4} \cdot 2 n^{1 / 3}$ of them) and into the shaded discs (there are $n^{1 / 4} \cdot n^{1 / 4}$ of them). The root of $F_{\sqrt{n}, \sqrt{n}}$ is embedded onto the bottom vertex of the whole triangle.

In the following, we describe the embedding in more detail. It will be done in two steps. In the first step, we embed vertices up to the level $h=2 n^{1 / 3}$ into the dashed trapezoids on the corresponding level. In the second step, we embed all the other vertices on the diameters of the discss and change the positions of some vertices on levels between $n^{1 / 3}$ and $2 n^{1 / 3}$.

Step 1. We start with the vertices on level $h=2 n^{1 / 3}$. There are $n^{1 / 2}$ vertices on this level. We divide them into $n^{1 / 4}$ groups, each of size $n^{1 / 4}$. Each of these groups is packed into one of the dashed trapezoids (on the last level). This is possible since the area of each trapezoid on this level is $\Omega\left(n^{11 / 12}\right)$, which is 


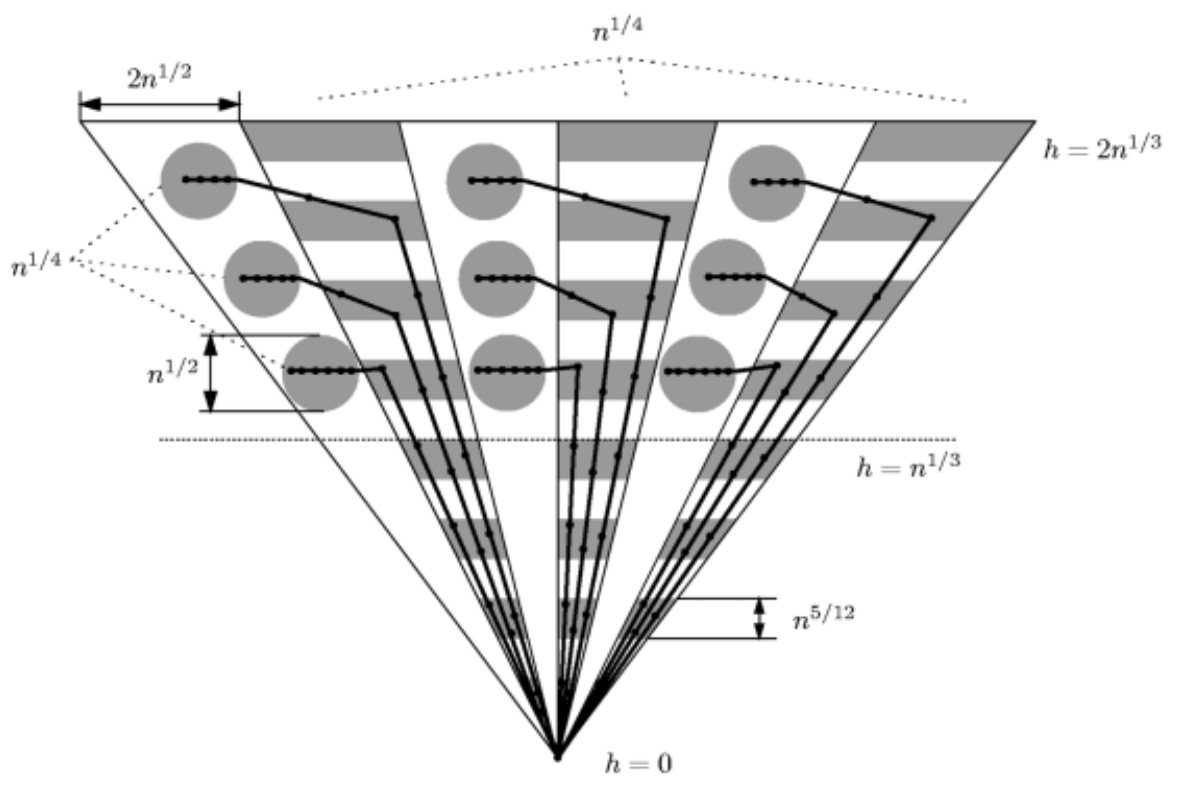

Fig. 2. The embedding of $F_{\sqrt{n}, \sqrt{n}}$.

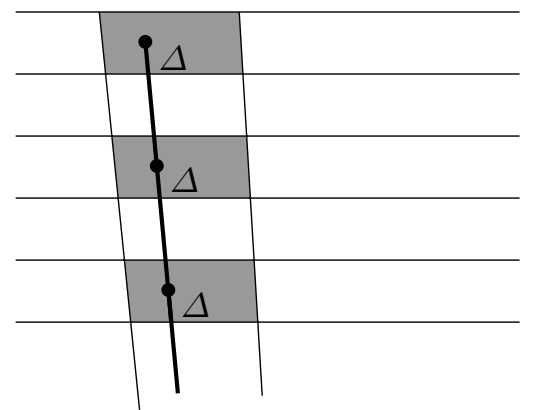

Fig. 3. The placement of vertices in lower levels.

exactly the required area (we have $n^{1 / 4}$ vertices, any two of them must be at distance at least $\Omega\left(n^{1 / 3}\right)$ ). When all the vertices on the level $h=2 n^{1 / 3}$ are embedded, we embed the vertices on lower levels. We connect every vertex $x$ on the last level by a straight line to the root. On this line we embed all the vertices on the path from $x$ to the root. We do this in such a way that the distance $\Delta$ (measured on the connecting line) from the vertex to the bottom of the dashed trapezoid is the same for all the vertices on the path from $x$ to the root (see Fig. 31). It is easy to check that the distance between the vertices on the last level guarantees that the vertices in the lower levels are also far enough from 
each other. Indeed, if we consider two vertices $x_{1}$ and $y_{1}$ at distance $d$ in level $h_{1}$ (i.e. their tree-distance is $2 h_{1}$ ) then for any $x_{2}$ on the path from $x_{1}$ to the root and any $y_{2}$ on the path from $y_{1}$ to the root, both in the level $h_{2}$, their distance is at least $d h_{2} / h_{1}$ (while their tree-distance is $2 h_{2}$ ).

Thus, the construction described so far gives an embedding of $F_{\sqrt{n}, 2 n^{1 / 3}}$ with distortion $O\left(n^{5 / 12}\right)$. In the next step we modify this embedding into an embedding of $F_{\sqrt{n}, \sqrt{n}}$.

Step 2. In this step we embed all the vertices on the remaining levels. We also change the positions of some vertices on the levels between $n^{1 / 3}$ and $2 n^{1 / 3}$. Left to the $n^{1 / 4}$ columns of trapezoids there are $n^{1 / 4}$ columns of discs (and there are $n^{1 / 4}$ discs in each column). In each of the discs one path ends. A more precise description follows.

We start from the bottom and continue upwards. For each disc we consider the level nearest to its equator (below the equator). We take the leftmost vertex in the shaded trapezoid on this level and change the embedding of its successors: all of its successors are embedded on the (possibly extended) equator of the considered disc. The distance between any two neighbours on this path is 1 in the embedding. We continue with the next higher level (with a smaller number of paths). In the last level, there is only one path to end. The resulting embedding is indicated in Fig 2,

It is straightforward to check that the resulting embedding of $F_{\sqrt{n}}, \sqrt{n}$ yields distortion $O\left(n^{5 / 12}\right)$ as claimed.

Acknowledgment. We would like to thank Helena Nyklová, Petra Smolíková, Ondřej Pangrác, Robert Šámal, and Tomáš Chudlarský for useful discussions on the problems considered in this paper.

\section{References}

1. Y. Bartal. Probabilistic approximation of metric spaces and its algorithmic applications. In Proc. 37th Ann. IEEE Sympos. on Foundations of Computer Science, pages 184-193, 1996.

2. U. Feige. Approximating the bandwidth via volume respecting embeddings. $J$. Comput. Syst. Sci, 60:510-539, 2000.

3. A. Gupta. Embedding tree metrics into low dimensional Euclidean spaces. Discrete Comput. Geom., 24:105-116, 2000.

4. N. Linial, E. London, and Yu. Rabinovich. The geometry of graphs and some its algorithmic applications. Combinatorica, 15:215-245, 1995.

5. J. Matoušek. Bi-Lipschitz embeddings into low-dimensional Euclidean spaces. Comment. Math. Univ. Carolinae, 31:589-600, 1990.

6. S. Rao. Small distortion and volume respecting embeddings for planar and Euclidean metrics. In Proc. 15th Annual ACM Symposium on Comput. Geometry, 1999. 